\title{
Assesment of Endometrial Vascularization with Immunohistochemical CD34 Assay in Recurrent Pregnancy Loss: A Case Control Study
}

\author{
Emine AYDIN', A. Taner USTA ${ }^{2}$ \\ Ankara, Turkey
}

\section{ABSTRACT}

OBJECTIVES: We compared the endometrial vascularization in hysteroscopic endometrial samplings between recurrent pregnancy loss (RPL) and control group.

STUDY DESIGN: We prospectively evaluated hysteroscopic endometrial samplings from recurrent pregnancy loss and control groups. CD34 transmembrane protein was used for evaluating endometrial vascularization. The vascularization was assessed based on thickness of vessels, diameter of the largest vessel, and number of vessels per $\mathrm{mm}^{2}$ in CD34-stained slides.

RESULTS: There was no significant difference in demographic findings and vascularization, such as largest vessel diameter (p: 0.572), and number of vessels per $\mathrm{mm}^{2}(\mathrm{p}: 0.982)$ between the two groups.

CONCLUSION: The cycling endometrium is a highly angiogenic tissue and may play a role in the etiology of RPL. However, we find a weak relationship between endometrial vascularization and recurrent pregnancy loss.

Keywords: Recurrent pregnancy loss, Vascularization, Endometrium, CD34

Gynecol Obstet Reprod Med 2017;23(2):70-73

\section{Introduction}

Recurrent pregnancy loss (RPL), a condition of three or more consecutive pregnancy losses before the pregnancy reaches 20 weeks, is observed in $1 \%$ of women in their reproductive age (1). Recently, RPL is defined as two consecutive pregnancy losses, a health problem that affects $5 \%$ of women. Many studies have been conducted to find an association between RPL and genetic, immune, infectious, and endocrine disorders (2-6). However, its etiology is not known in more than $50 \%$ of the RPL cases (2). In this study, we investigate the con-

${ }^{1}$ Hacettepe University Faculty of Medicine Department of Obstetrics and Gynecology, Ankara

${ }^{2}$ Department of Obstetrics and Gynecology Bağcllar Education and Research Hospital, Istanbul

Address of Correspondence: Emine Aydin

Hacettepe University, Faculty of

Medicine Department of Obstetrics and

Gynecology, Division of Maternal Fetal

Medicine, Sihhiye, Ankara, Turkey

eminebaskurtaydin@gmail.com

Submitted for Publication: $\quad 02.09 .2016$

Accepted for Publication: $\quad 09.02 .2017$

\begin{tabular}{|c|c|}
\hline \multicolumn{2}{|c|}{ Access this article online } \\
\hline 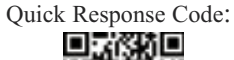 & Website: www.gorm.com.tr \\
\hline 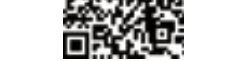 & DOI:10.201613/GORM.2016.664 \\
\hline
\end{tabular}

How to cite this article: Aydin E. and Usta AT. Assesment of Endometrial Vascularization with Immunohistochemical CD34 Assay in Recurrent Pregnancy Loss: A Case Control Study. Gynecol Obstet Reprod Med 2017;23(2):70-3 tribution of vascularization to RPL (6). Endometrial tissue is regenerated dynamically and continuously during menstrual cycle, making vascularization essential to the endometrium. Previous studies have investigated vascularization in decidua and placenta in RPL cases and its indirect association with various factors $(7,8)$. The main purpose of the present study is to compare endometrial angiogenesis/vascularization in RPL and healthy fertile women. To analyze the endometrial vascularization in non-pregnant RPL cases, we used CD34, which is a transmembrane protein (9). It was previously used for evaluation of vascularization in decidua and comparison of parietal and basal decidua vascularization $(7,10)$. In this study, we aim to demonstrate the effect of vascularization in RPL.

\section{Material and Method}

\section{Selection of patients and inclusion and exclusion criteria}

Patients with a history of RPL, wishing to have a child, and referred to the outpatient clinic of Obstetrics and Gynecology Department of a tertiary research hospital were included in the study. Recurrent abortion was considered as at least 2 or more consecutive spontaneous pregnancy loses before 20 weeks of gestation (11). The controls were healthy women referred to the outpatient clinic for annual routine gynecologic control during the same period. Women with a history of abortion, irregular menstruation, and who received hormonal therapy 3 months prior to biopsy were excluded. (Figure 1). Patients incidentally detected with an endometrial pathology, such as polyp, in office hysteroscopy examination were excluded from the study. Women who had an abortion at 
least 4 months before were included in the patient group. For all patients selected in the study, sexual intercourse after the last menstrual period was forbidden.

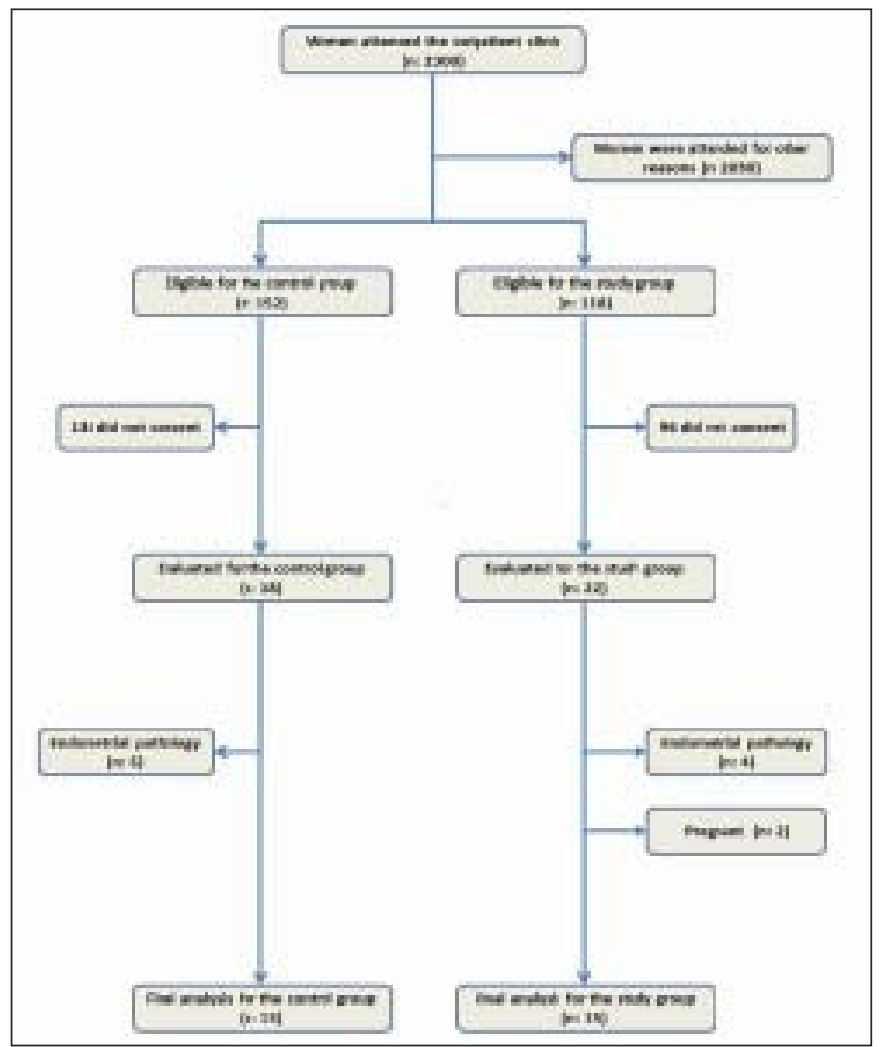

Figure 1: Patient selection

\section{Preparation of tissue specimens}

Tissue specimens were taken during office hysteroscopy using hysteroscopic forceps and a rigid standard hysteroscopy (Karl Storz) was used for the procedure. The endometrial biopsy specimens were taken during the luteal phase of menstrual cycle.

This study was approved by the institutional ethics review board (reference number: 2012/33) and registered with ClinicalTrials.gov (identifier: NCT01735331). Oral and written information was given to the participating patients; subsequently, their written informed consent was taken.

\section{Histopathological assessment}

The tissue specimens were fixed in 10\% formalin and embedded in paraffin. Sections of $4-5-\mu \mathrm{m}$ thickness were processed for microscopic slides. The slides were stained with hematoxylin and eosin. For immunohistochemical assays, the sections were passed through alcohol and xylol protocol at room temperature for deparaffinization. The sections were kept in $0.3 \% \mathrm{w} / \mathrm{v} \mathrm{H}_{2} \mathrm{O}_{2}$ in distilled water and washed with PBS for 3-5 min (PBS: Phosphate buffered saline, $\mathrm{pH} 7.2$ - 7.4). The sections then underwent antigen retrieval procedure with citrate buffer ( $\mathrm{pH} \mathrm{6)}$ in a microwave oven at medium setting for $15 \mathrm{~min}$. Subsequently, the chalet with closed lid was cooled at room temperature for $45 \mathrm{~min}$. Then, the sections were passed through distilled water and PBS. The sections were incubated with normal blocking serum for 8-10 min; subsequently, the solution on the tissue was discarded and area around the tissue was wiped dry. Primary antibody binding was determined for $\mathrm{CD} 34$; the reaction was revealed with $\mathrm{DAB}$, and the slides were counterstained with Mayer's hematoxylin and mounted.

\section{Evaluation of vascularization}

A single experienced pathologist who was unaware if patients were from RPL or control group performed all pathological assessments. A microscope (BX51, Olympus America, Inc., Melville, New York) was used to examine the tissue sections; 4-6 tissue sections from each patient were examined for evaluation. In each section, eight fields with 200x magnification were studied. The vascularization was evaluated according to the thickness of vessels, diameter of the largest vessel, and the number of vessels per $\mathrm{mm}^{2}$ in CD34-stained slides (Figure 2 and 3).

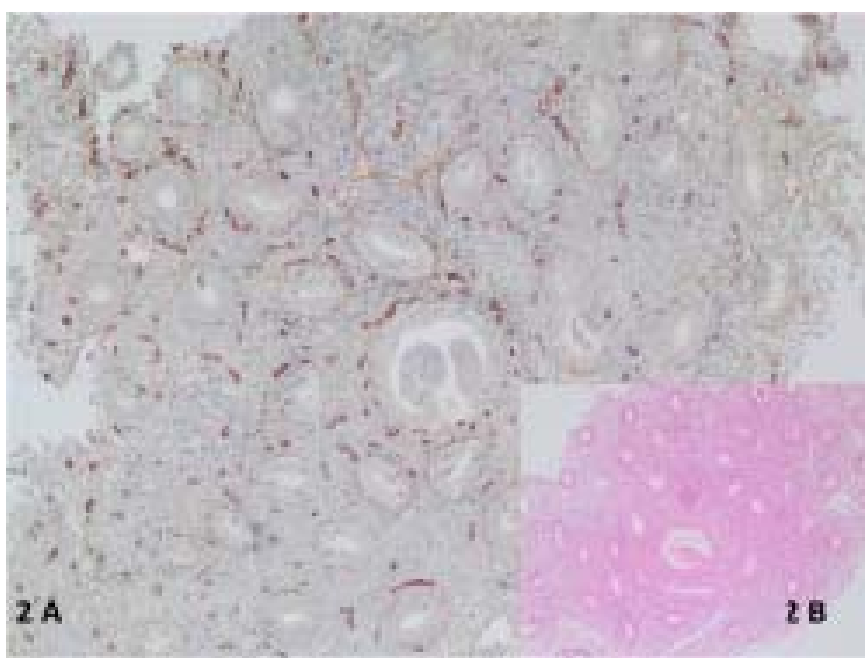

Figure 2A: CD34 staining revealed vascular structure of endometrium (x10), B: H\&E stain section of same slide (x10).

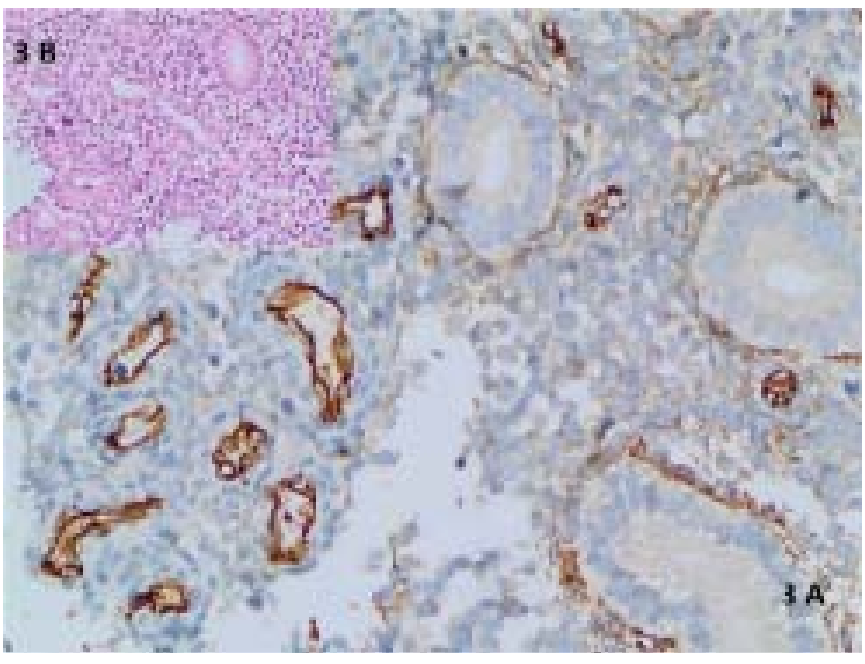

Figure 3A:Thick wall vessels (CD34 stained $x 40)$, the vascularization was evaluated according to the thickness of vessels in CD34-stained slides, B: H\&E stained section of same slide (x40). 


\section{Statistical analysis}

Statistical analysis was performed using Statistical Package for the Social Sciences v.18.0 for Windows (SPSS, Inc., Chicago, IL). Variables were investigated by using visual and analytical methods (Kolmogorov-Smirnov test/Shapiro Wilk's test) to determine their normal distribution. The Student's t-test was used to compare the normally distributed variables. Because diameter of the large vessels was not normally distributed, Mann-Whitney U test was used to compare the groups. Chi-square test or Fisher's exact test was appropriately used to compare the proportions in case and control groups. A p-value of 0.05 was considered as statistically significant.

\section{Results}

Age, number of gravidity, and abortions in the patient and control groups are summarized in table 1. There was no abortion in the control group. In the RPL group, median number of abortions was three, and its interquartile range was 2-4. There was no difference in age and number of gravidity between the case and control groups. Pregnancies of all women in the control group ended in healthy births. In the RPL group, the thickness of vessels per magnification field was $53.3 \%$, the diameter of the largest vessel was $0.16 \pm 0.6 \mathrm{~mm}$, the vessel count per magnification field was 30 (range: 47-22); there was no significant difference in vascularization between the two groups (Table 2).

\section{Discussion}

The aim of this study was to compare endometrial vascularization of women in RPL and control groups with successful pregnancies. Our results show that there was no difference in endometrial vascularization between the two groups.

A previous study compared endometrial vascularization in healthy fertile women in RPL cases using 3-dimensional Doppler angiography, and reported a significant decrease in vascularization in the RPL cases (12). Similarly, another indirect comparative study by Doppler angiography reported an impairment of endometrial vascularization in antiphospholipid antibody-positive RPL cases (13). In the present study, we directly assessed vascularization in the tissue. Our comparison was optimal because number of pregnancies in both the groups was equal, patients had regular menstrual periods, and we applied strict criteria to avoid other factors that could affect vascularization.

Direct assessment of endometrial vascularization was previously conducted by two studies. One study involved histopathological evaluation of decidual vascularization in women with missed abortion that were diagnosed by sonographic examination and clinical findings (8). The control group consisted of women with viable intrauterine pregnancy referred for medical abortion, and the decidual vascularization in both the groups was compared. The prognosis of pregnancies in the control group could not be foreseen. The prevalence of abortion in normal populations is $8 \%-20 \%$ (14) and some patients in the control group could have suffered abortion in the absence of medical abortion. This makes the control group unreliable; thereby, rendering the study imperfect.

In the other study, vascularization in endometrial pipelle biopsy material of women with a history of RPL was compared with vascularization in hysterectomy specimens of controls (8). For optimal evaluation, it is essential that the patients have regular menstrual periods. The possible presence of irregular menstruation or menopause in the control group, and higher mean age of the hysterectomy group exposes limitations in the study. In contrast, the subjects in the patient and control groups in our study were selected almost perfectly.

Table 1: Demographic features of the patients

\begin{tabular}{lccc}
\hline & RPL group $(\mathrm{n}=15)$ & Control group $(\mathrm{n}=13)$ & $\mathrm{p}$ value \\
\hline Age & $31.4 \pm 6.8$ & $35.6 \pm 7.6$ & $0.137^{\mathrm{a}}$ \\
Gravidity & $4(3.0-5.0)$ & $3(4.5-2.0)$ & $0.156^{\mathrm{b}}$ \\
Live birth & $1(1.0-0.0)$ & $3(4.5-2.0)$ & $<.000^{\mathrm{b}}$ \\
\hline
\end{tabular}

RPL: Recurrent pregnancy loss, Row data are mean \pm standard deviation, a: Student's $t$ test; Row data are median (interquartile range), b: MannWhitney Rank Sum test

Table 2: Comparison of vasculogenesis in the recurrent pregnancy loss and control groups

\begin{tabular}{lccc}
\hline & RPL group $(n=15)$ & Control group $(n=13)$ & $p$ value \\
\hline Largest vessel diameter & $0.16 \pm 0.6$ & $0.14 \pm 0.9$ & $0.572^{\mathrm{a}}$ \\
Thick vessel count per magnification field & $53.3 \%$ & $23.1 \%$ & $0.102^{\mathrm{b}}$ \\
Vessel count per magnification field & $30(47-22)$ & $28(22.5-40.5)$ & $0.982^{\mathrm{c}}$ \\
\hline
\end{tabular}

RPL: Recurrent pregnancy loss, Row data are mean \pm standard deviation a: Student's t test, Row data are proportion, b: Chi-square test, and Row data are median (interquartile range), c: Mann-Whitney Rank Sum test 
A major limitation of our study is the limited number of patients included. This was because of the strict criteria of patient selection. In addition, some subjects in the control group refused the invasive methods of hysteroscopy and biopsy because they showed no pathological finding and had no history of abortion. Nevertheless, we could make statistically significant evaluation. Future studies on etiologic factors other than endometrial vascularization or different methods of assessing vascularization are required. Different studies continue to be carried out in the literature that address this issue.

\section{References}

1. Stirrat GM. Recurrent miscarriage. Lancet 1990;336 (8716):673-5

2. Jeve YB, Davies W. Evidence-based management of recurrent miscarriages. J Hum Reprod Sci 2014;7(3):159-69.

3. de Jong PG, Goddijn M, Middeldorp S. Testing for inherited thrombophilia in recurrent miscarriage. Semin Reprod Med 2011;29(6):540-7.

4. Rote NS. Antiphospholipid antibodies other than lupus anticoagulant and anticardiolipin antibodies in women with recurrent pregnancy loss, fertile controls, and antiphospholipid syndrome. Obstet Gynecol 1997;90(4 Pt 1):642-4.

5. Fuhrer D, Mann K, Feldkamp J, Krude H, Spitzweg C, Kratzsch J, et al. Thyroid dysfunction in pregnancy. Dtsch Med Wochenschr 2014;139(42):2148-52.

6. Su MT, Lin SH, Lee IW, Chen YC, Kuo PL. Association of polymorphisms/haplotypes of the genes encoding vascular endothelial growth factor and its KDR receptor with recurrent pregnancy loss. Hum Reprod 2011;26(4):758-64.

7. Plaisier M, Dennert I, Rost E, Koolwijk P, van Hinsbergh
VW, Helmerhorst FM. Decidual vascularization and the expression of angiogenic growth factors and proteases in first trimester spontaneous abortions. Hum Reprod 2009; 24(1):185-97.

8. Lash GE, Innes BA, Drury JA, Robson SC, Quenby S, Bulmer JN. Localization of angiogenic growth factors and their receptors in the human endometrium throughout the menstrual cycle and in recurrent miscarriage. Hum Reprod 2012;27(1):183-95.

9. Sutherland DR, Keating A. The CD34 antigen: structure, biology, and potential clinical applications. J Hematother 1992;1(2):115-29.

10. Thorin E, Shatos MA, Shreeve SM, Walters CL, Bevan JA. Human vascular endothelium heterogeneity. A comparative study of cerebral and peripheral cultured vascular endothelial cells. Stroke 1997;28(2):375-81.

11. Ford HB, Schust DJ. Recurrent pregnancy loss: etiology, diagnosis, and therapy. Rev Obstet Gynecol 2009;2(2):7683.

12. Chen L, Quan S, Li H, Chen C, Xing F, Yu Y. A comparison of endometrial and subendometrial vascularity assessed by three-dimensional ultrasonography and power Doppler angiography between healthy fertile women and women with unexplained primary recurrent miscarriage. Fertil Steril 2011;95(3):1127-9.

13. Chen L, Quan S, Ou XH, Kong L. Decreased endometrial vascularity in patients with antiphospholipid antibodiesassociated recurrent miscarriage during midluteal phase. Fertil Steril 2012;98(6):1495-502 e1.

14. Wilcox AJ, Weinberg CR, O'Connor JF, Baird DD, Schlatterer JP, Canfield RE, et al. Incidence of early loss of pregnancy. N Engl J Med 1988;319(4):189-94. 